\author{
Magdalena Dutsch-Wicherek ${ }^{1}$, Romana Tomaszewska², Agata Lazar ${ }^{2}$, Konrad Dziobek $^{3}$, \\ Przemko Kwinta ${ }^{4}$, Łukasz Wicherek ${ }^{5}$ \\ ${ }^{1}$ Department of Paediatric Otolaryngology, Chair of Paediatrics, Jagiellonian University Medical College, Kraków, Poland \\ 2Department of Pathology, Jagiellonian University, Kraków, Poland \\ ${ }^{3}$ Lukaszczyk Oncological Centre in Bydgoszcz, Poland \\ ${ }^{4}$ Chair of Paediatrics, Jagiellonian University Medical College, Kraków, Poland \\ ${ }^{5}$ Chair of Oncology, Radiotherapy and Gynaecological Oncology, Ludwik Rydygier Collegium Medicum in Bydgoszcz, \\ Nicolaus Copernicus University, Bydgoszcz, Poland
}

\title{
Metallothionein immunoreactivity profile in B-cell lymphomas of the palatine tonsils
}

\author{
Corresponding author: \\ Dr. hab. n. med. \\ Magdalena Dutsch-Wicherek \\ Department of Paediatric \\ Otolaryngology, Chair of Paediatrics \\ Jagiellonian University Medical College \\ Wielicka 265 St., 30-663, Kraków \\ Poland \\ E-mail: \\ magdalena.dutsch-wicherek@uj.edu.pl
}

Medical Research Journal 2017; Volume 2, Number 2, 37-45 10.5603/MRJ.2017.0007 Copyright (C) 2017 Via Medica ISSN 2451-2591

\begin{abstract}
Introduction. Tumours stimulate the remodelling of their microenvironment for their own survival. To protect their own growth and induce angiogenesis, tumours change the structure of the extracellular matrix and alter the function of existing as well as chemo-attracting immune system cells. MT is an anti-apoptotic and pro-proliferative protein that is also responsible for modulating the response of immune system cells. The expression of this protein by the fibroblasts of the tumour microenvironment is probably related to the remodelled phenotype of these cells by tumour influence on cancer-associated fibroblasts. Vimentin is a protein that appears to be the marker for the mesenchymal transition of cells from the epithelial phenotype. These cells seem to acquire the mesenchymal phenotype so that they can migrate and facilitate the development of metastases. Interestingly, the expression of vimentin has also been observed in the tumour microenvironment and may serve as a marker of a remodelled stroma in the process of facilitating tumour spread.

Materials and methods. We recruited 25 patients with tonsillar DLBCL (diffuse large B-cell lymphoma) and tonsillar DLBCL with cervical lymph node involvement (i.e. stages I and II of the disease) and analysed tissue samples from the lymphoma and tumour microenvironment of each. We also analysed the immunoreactivity levels of the following antigens in the palatine tonsil lymphoma and its stroma: MT, vimentin, and CD56- and CD57-positive cells.

Results. A statistically significantly higher MT and vimentin immunoreactivity was observed in the lymphoma as compared to the stroma tissue samples. However, both MT-positive fibroblasts and MT-positive macrophages were observed in the stroma. Additionally, statistically significantly lower numbers of CD56and CD57-positive cells were identified in the lymphoma and the stroma samples than in the reference group samples.

Conclusions. The high vimentin immunoreactivity in the tumour and its stroma, together with MT-expressing fibroblasts and macrophages, as well as a CD56- and CD57-positive cell deficit, would seem to confirm microenvironment remodelling and the participation of MT in tumour remodelling.

Key words: MT, tumour microenvironment, vimentin, tumour-associated macrophages, cancer-associated fibroblasts, EMT
\end{abstract}

Med Res J 2017; 2 (2): 37-45

\section{Introduction}

Malignant lymphoma is a disease originating primarily from the lymph nodes; however, $24-48 \%$ of all non-Hodgkin lymphomas (NHL) may arise from extranodal sites. Over the past few decades, the incidence of such types of lymphomas has increased [1]. It is estimated that $10 \%$ of patients with NHL present with extranodal disease in the head and neck region [2]. While more than half of the head and neck lymphomas occur in the Waldeyer's ring [2, 3], about $40-50 \%$ arise in the palatine tonsils $[4,5]$. The majority of NHL arising 
from the tonsils are diffuse, large B-cell lymphomas (DLBCL) [6, 7].

Metallothioneins are low-molecular weight proteins with a high affinity for divalent metals such as zinc and copper, as well as toxic metal ions such as cadmium and mercury $[8,9]$. The ability to bind the metal ions is linked to the biological role of these proteins, which includes protection against metal toxicity, the reservoir of zinc and copper to metalloenzymes during the apoptosis process, the production of transcription factors, and protection against oxidative stress [8]. MTs may also play an important role in the proliferation and differentiation of cells [10]. Moreover, it has been established that MT expression in the cytoplasm helps to protect against cytotoxicity, while its expression in the nucleus protects against genotoxicity $[8,10,11]$. Genotoxicity concerns the acquisition of cells of the malignant phenotype, as a result of mutations critical to the carcinogenesis. Cytotoxicity is important in the interaction of cancer cells with immune system cells. MT expression has been observed in various types of malignant neoplasms and in cancer microenvironments as well as in healthy tissues adjacent to cancer nests [10-12]. As has been demonstrated in various studies, MT expression in healthy epithelia is localised in the basal part of the epithelium, which comprises intensively dividing cells responsible for its renewal, while in the more superficial layers of the epithelium, which are composed of well-differentiated cells, MT expression has not been found [13-18]. MT expression has also been observed in tumour-adjacent tissue, epithelium, and even in tumours without MT expression [13-20].

The tumour microenvironment is the tissue that supports the tumour's growth [21]. In our previous studies, we demonstrated and analysed the involvement of the tumour microenvironment in the development of various types of malignant epithelial neoplasms. The microenvironment was associated with its own remodelling and the acquisition of a suppressive tumour microenvironment [22-25]. Additionally, we demonstrated the presence of tumour microenvironment remodelling with RCAS1 expression and RCAS1-positive macrophages in the tumour microenvironment of palatine tonsil DLBCL. RCAS1 also seemed to be involved in creating tumour-induced inflammation within the tumour and its microenvironment [26].

Vimentin is a $57 \mathrm{kDa}$, cytoskeletal protein involved in embryonic development. In adults, vimentin expression is reduced and is mainly observed in connective tissue mesenchymal cells in the CNS and in muscles [27-34]. Vimentin is expressed in a wide variety of cells, including trophoblastic giant cells, fibroblasts, macrophages, neutrophils, and leukocytes $[11,28]$. The most important role of vimentin is related to its involvement in the epithelial-mesenchymal transition (EMT) process, typified by its expression in epithelial cells, which normally express only keratin. Physiologically, this process is observed during embryonic development, wound healing, and chronic inflammation; in pathology metastasising cells demonstrate processes similar to EMT. This is significant because EMT has been associated with tumour progression [35-37]. Moreover, vimentin and MT-1 promoters have recently been shown to be epigenetically suppressed by PU.1, a transcription factor for haematopoiesis that plays important roles in haematological malignancies [38].

In the present study, we aimed to determine the immunoreactivity of MT and vimentin in the tumour and its microenvironment in palatine tonsils from which DLBCLs have originated.

\section{Materials and methods}

For our study, we recruited patients with the most common forms of non-Hodgkin lymphoma occurring in adults, namely, tonsillar DLBCL (diffuse large B-cell lymphoma) and tonsillar DLBCL with cervical lymph node involvement (i.e. stages I and II of the disease). We selected 25 patients and analysed tissue samples from the lymphoma and tumour microenvironment of each. The tumour microenvironment or stroma was defined as the surrounding tissue with an area of $1 \mathrm{~cm}^{2}$ macroscopically and histologically free of malignant infiltration, and with the distance from the tumour front not exceeding $1 \mathrm{~cm}$. The patient's consent was obtained in each case. Additionally, approval for the research program was granted by the Ethical Committee of the Jagiellonian University in Krakow (KBET/90/B/2005). All the tissue samples were histopathologically verified. Following the fixation of the surgically removed material in formalin, pathological analysis with classical haematoxylin and eosin staining techniques was performed in the Pathology Department of the Jagiellonian University by two experienced pathologists (R.T. and A.L.) working independently. The tissue was first fixated in a solution of $10 \%$ formalin, then rinsed, dehydrated, and transferred through a progressively increasing concentration of ethanol (from $50 \%$ to absolute alcohol), and a series of xylenes (I-III) to molten paraffin wax. Finally, the tissue blocks were sectioned and the resulting sections placed onto glass slides. The process was mainly automated; however, both the paraffin embedding and the cutting of the tissue samples into 3- to $4-\mu \mathrm{m}$-thick sections were done manually (Tab. 1).

\section{Reference group}

As a reference group, we chose to collect palatine tonsils that had been removed from patients suffering 
Table 1. The characteristics of the patient group

\begin{tabular}{lc}
\hline \multicolumn{2}{l}{ Lymphoma malignum non-Hodgkin B cellular } \\
\hline Patients & 25 \\
M & 15 \\
W & 10 \\
Age (range) average & $16-84(55.6)$ \\
M & $43-81(63.8)$ \\
W & $16-84(59.4)$ \\
\hline
\end{tabular}

Table 2. The characteristics of the reference group

\begin{tabular}{lc}
\hline Palatine tonsils & \\
\hline Number of patients & 20 \\
M & 12 \\
W & 8 \\
Age (range) average & $14-56(32.6)$ \\
M & $14-56(34.41)$ \\
W & $21-54(29.87)$ \\
\hline
\end{tabular}

from recurrent tonsillitis. In these tissue samples, we evaluated both the epithelium lining of the tonsils and the lymphoid tissue (Tab. 2).

\section{Immunohistochemical analysis}

In the present study, we analysed the immunoreactivity levels of various antigens in the palatine tonsil lymphoma and its stroma. We also aimed to evaluate the distribution of the antigen immunoreactivity throughout the tissue of the tumour, including its stroma. For this reason, we chose the immunohistochemical method for our study. This is also the only method that shows the actual architecture of the interaction between the tumour and its stroma. From each tumour, 3-4 tissue samples were taken. Each sample was embedded in paraffin and formed into a tissue block, which was then cut into sections. All the tissue sections were further histopathologically verified and immunohistochemistry was performed. Two observers, working independently and having no knowledge of the clinicopathological data, reviewed the immunohistochemical expression of MT and vimentin as well as of CD68, CD56, and CD57 antigens. Immunohistochemical analysis was performed in the Pathology Department of the Jagiellonian University. Five-micrometre slides from each case were stained to visualise the expression of MT-, vimentin-, and CD68-, CD56-, and CD57-positive cells. In all cases, the immunohistochemistry was performed applying the Envision method using a Dako Autostainer. The samples were stained automatically with immunohistochemical staining based on the antigen-antibody reaction. Microscopy was performed with an Axio Zeiss microscope, and the tissue sections were then evaluated under both $20 \times$ and $40 \times$ magnification. The following antibodies were applied: CD56, CD57 (Novocastra; dilution 1:50), vimentin (DAKO, Denmark; dilution 1:50), CD68 (DAKO, clone PG-M1; dilution 1:50), and MT (ABCAM; dilution $1: 25)$ for 10 minutes at room temperature. Visualisation of the reaction products was then performed using $A E C$ (3-amino-9-ethyl-carbazole) as a chromogen (AEC Substrate Chromogen ready-to-use, DAKO, Denmark) for 10 minutes at room temperature. Sections were counterstained with haematoxylin and mounted in glycergel. As a positive control, a tonsil specimen was taken for MT immunostaining. All stainings were performed with the same procedure but with the omission of the primary antibody as a negative control. Expression for MT and vimentin was evaluated in entire sections, both in the area of the tumour and in the stroma, as follows: 0 - no reactivity; +1 - weak, when any (also granular in paranuclear region) cytoplasmic staining pattern observed (in up to $10 \%$ of positive cells); +2-marked cytoplasmic (sometimes together with membranous staining in $11-30 \%$ of the cells); and $+3-$ high expression (more than $30 \%$ of positive cells). Variable scales were used to evaluate an amount of the cells semi-quantitatively, depending on their general number in the specimen. CD56+, CD57+, and CD68+ cells were thus estimated as follows: 0 - lack of positive cells; +1 - single positive cells in the specimen; $+2-1-5$ positive cells/HPF; and $+3-$ more than five positive cells/HPF.

\section{Statistical analysis}

The distribution of variables in the study groups of women, checked with the use of the Shapiro-Wilk test, showed that each of the women was different from normal. The statistical significance between the groups was determined by the Kruskal-Wallis test, one-way analysis of variance by ranks. The Mann-Whitney $U$ test was then used as applicable. All statistical analyses were carried out with the Statistica 8.0 software program. A p value $<0.05$ was considered indicative of statistical significance.

\section{Results}

The immunoreactivity of the various antigens in the DLBCL and stromal tissue samples are discussed below.

\section{MT immunoreactivity in the lymphoma samples and the samples from the reference group}

MT immunoreactivity was observed in $70 \%$ of the lymphoma tissue samples and in $45 \%$ of the stroma tissue samples and represented the nuclear-cytoplasmic type of expression. In the stroma samples, MT immunoreactivity was observed in stromal fibroblasts (Fig. 1). 

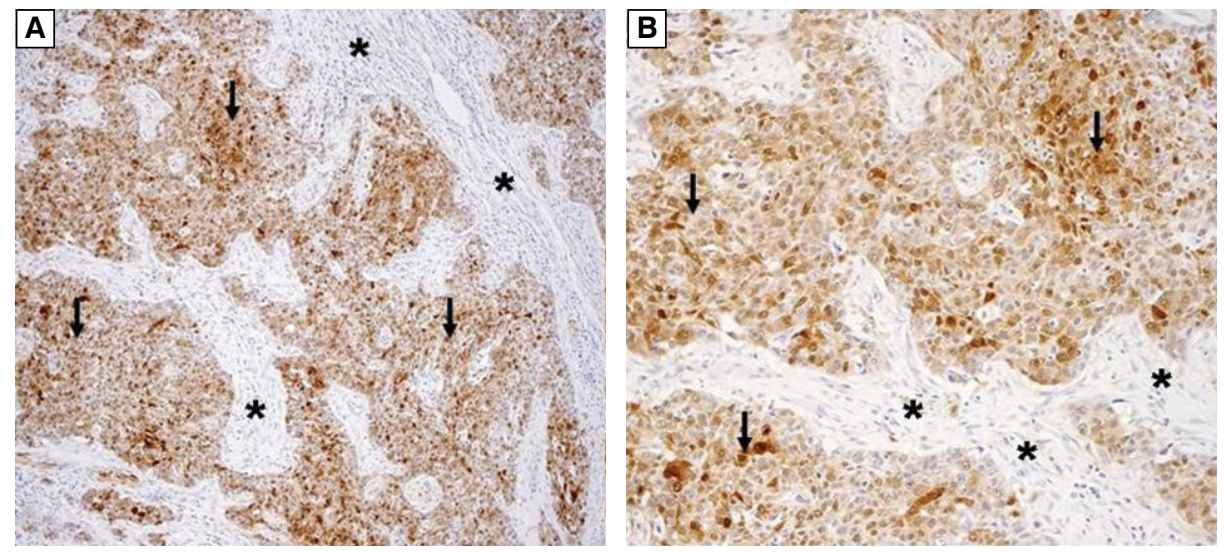

Figure 1. MT immunoreactivity in the lymphoma of palatine tonsils (arrows) and single fibroblasts in the stroma with MT immunoreactivity (stars). A - magnification 20×, B - magnification 40×

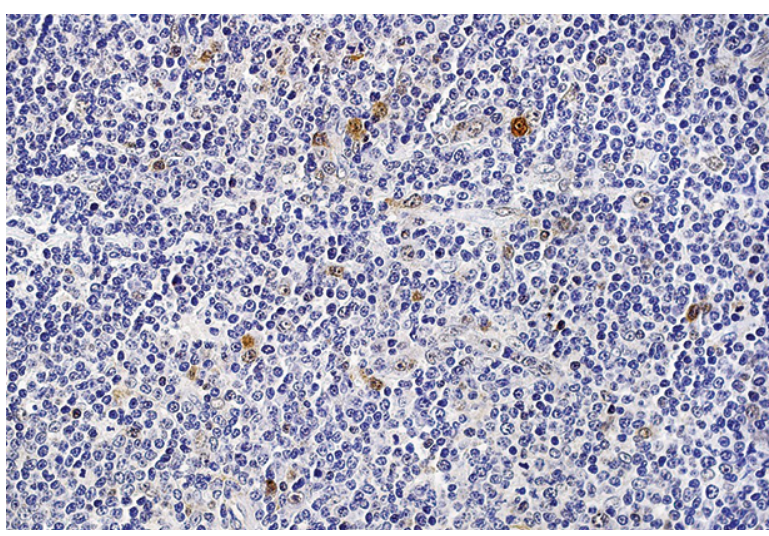

Figure 2. MT immunoreactivity in lymphoma cells and macrophages, magnification $40 \times$

In the lymphoma tissue samples, MT was observed in both lymphoma cells and in tumour-infiltrating macrophages (Fig. 2).

In the reference group, MT immunoreactivity was observed in both the lymphatic tissue and the epithelium lining the tonsils. It was present in $85 \%$ of the lymphatic tissue samples and was represented by dispersed spindle-shaped cell-fibroblasts. It was also present in $90 \%$ of the epithelium tissue samples where the immunoreactivity was present in the lower $1 / 3$ of the epithelium lining the tonsils and represented the membrane-cytoplasmic type of expression (Fig. 3). The most prominent MT immunoreactivity was observed in the epithelium of tonsillar crypts.

Vimentin immunoreactivity in the lymphoma samples and the samples from the reference group

Vimentin immunoreactivity was present in all the lymphoma tissue samples and in $80 \%$ of the stroma tissue

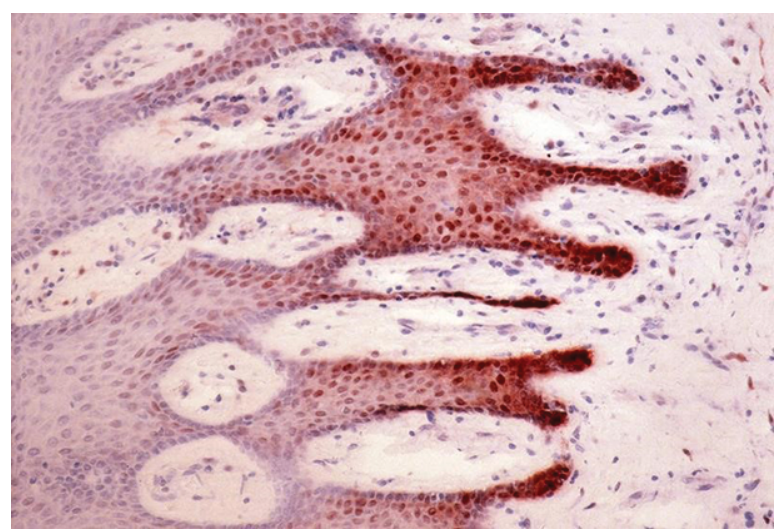

Figure 3. MT immunoreactivity in the basal layer of epithelium of palatine tonsils crypts

samples and represented a membranous-cytoplasmic pattern of expression (Fig. 4).

Strong vimentin immunoreactivity was observed in the tissue of the palatine tonsils from patients with chronic tonsillitis and in all the samples of lymphatic tissue; vimentin immunoreactivity was not observed in the epithelium lining the tonsils.

\section{CD56 immunoreactivity in the lymphoma samples and the samples from the reference group}

CD56-antigen immunoreactivity was detected in $10 \%$ of the lymphoma tissue samples and in $20 \%$ of the stromal tissue samples and represented a membranous-cytoplasmic type of expression (Fig. 5). CD56-antigen immunoreactivity was observed in $30 \%$ of the lymphatic tissue samples of the palatine tonsils from patients with chronic tonsillitis and represented the membranous-cytoplasmic type of expression; it was not observed in the epithelium lining the tonsils (Fig. 5). 

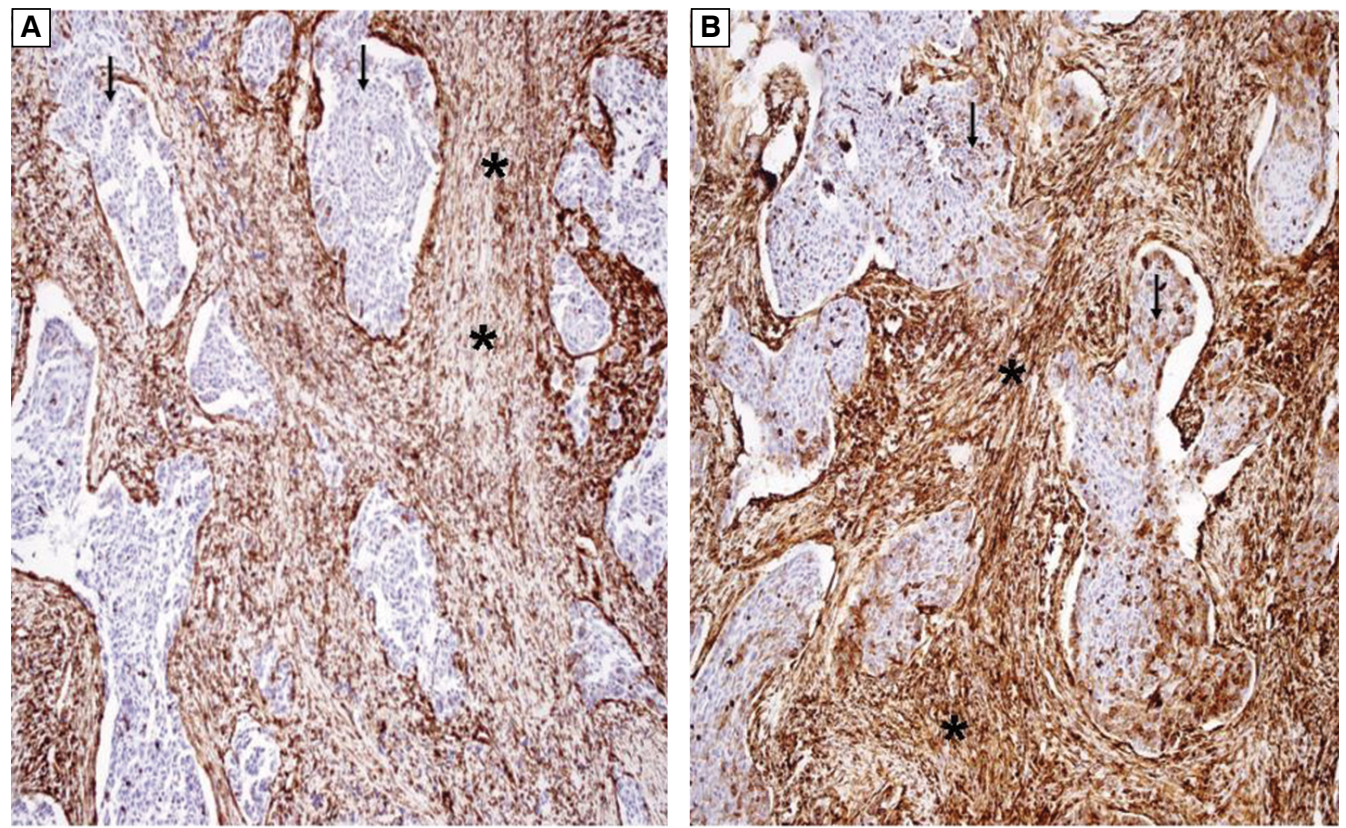

Figure 4. Vimentin immunoreactivity in the lymphoma of palatine tonsils and its stroma. A - strong immunoreactivity in the stroma, the lymphoma tissue is surrounded by stroma with strong vimentin immunoreactivity (magnification 20X) - stars, with a weak immunoreactivity in the tumour tissue-arrows; B - strong immunoreactivity in the stroma - arrows and strong immunoreactivity in the peripheral part of tumour nests (magnification $20 \times$ ). Strong vimentin immunoreactivity was observed in the tissue of palatine tonsils under chronic tonsillitis, in all the tissue samples of lymphatic tissue, while vimentin immunoreactivity was not observed in the epithelium lining the tonsils
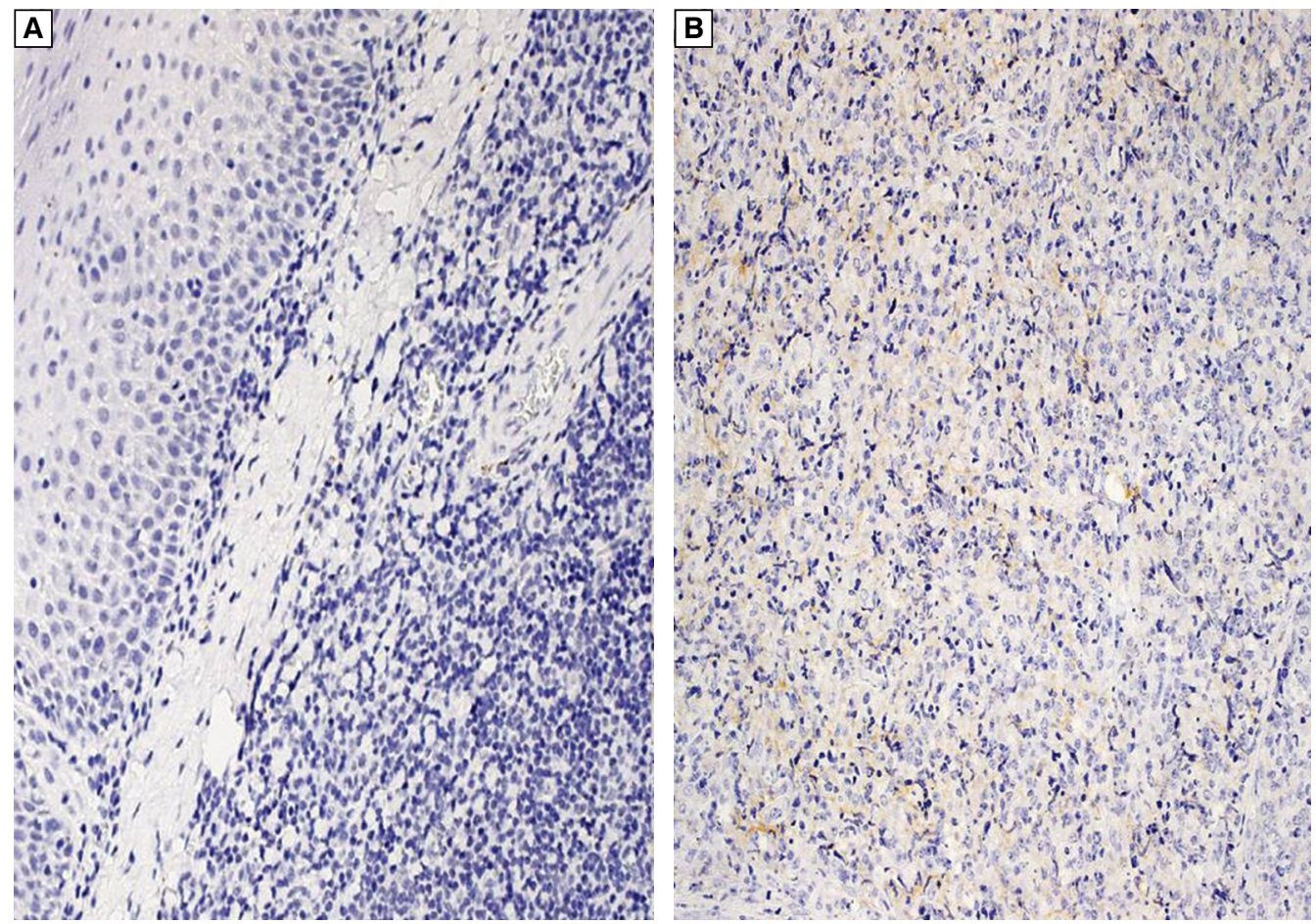

Figure 5. Weak CD56 immunoreactivity in the lymphoid tissue of palatine tonsils of chronic tonsillitis (A) and in lymphoma tissue samples (B) 

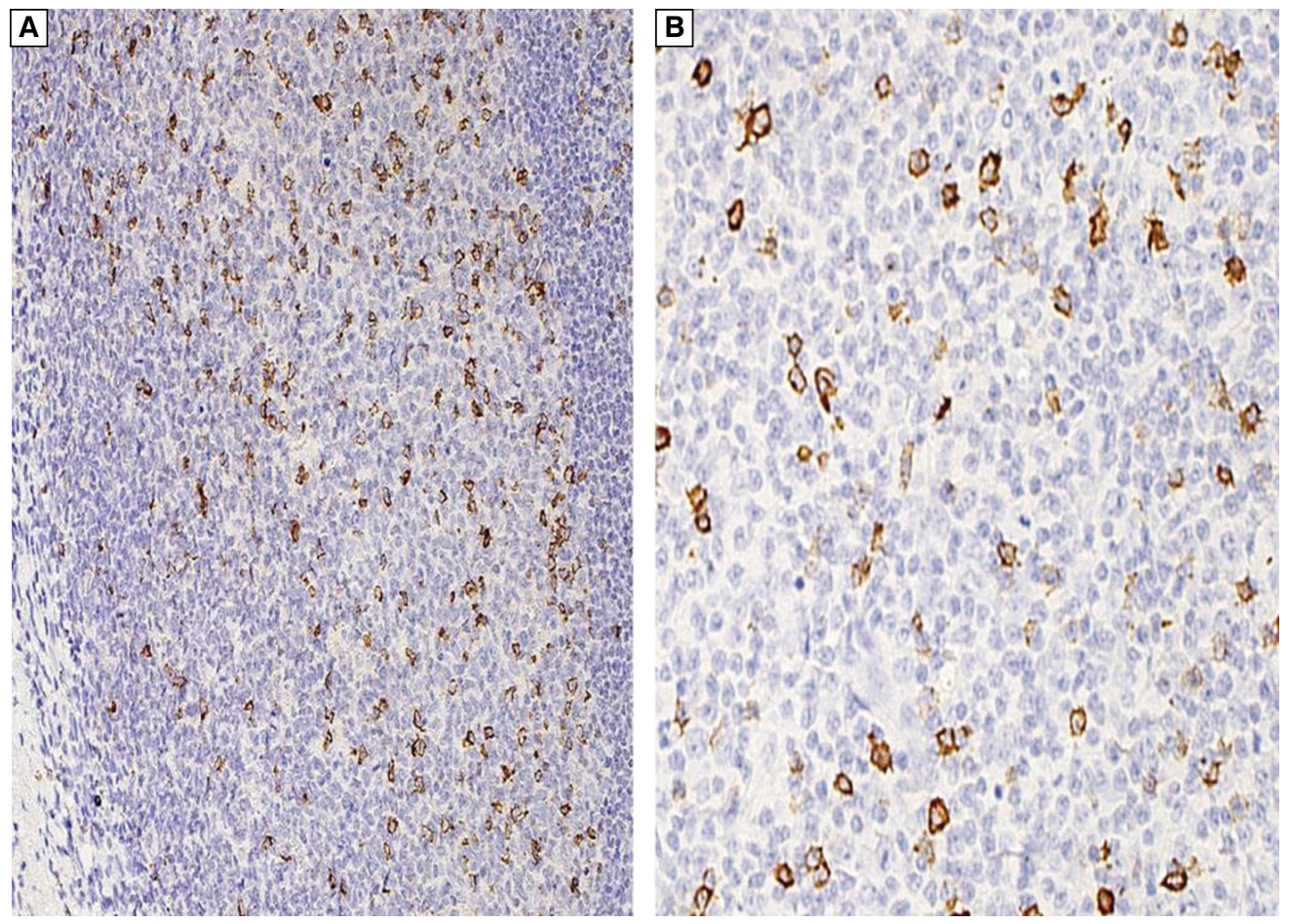

Figure 6. CD57 antigen immunoreactivity in the tissue of palatine tonsils (A) and in the lymphoma (B)

Table 3. The comparison of analysed antigen immunoreactivity in the tissue of the lymphoma and its stroma

\begin{tabular}{lccc}
\hline Antigen & $\begin{array}{c}\text { Lymphoma } \\
\text { Median }\left(\mathbf{Q}_{\mathbf{3}}-\mathbf{Q}_{\mathbf{1}}\right)\end{array}$ & $\begin{array}{c}\text { Stroma } \\
\text { Median }\left(\mathbf{Q}_{\mathbf{3}}-\mathbf{Q}_{\mathbf{1}}\right)\end{array}$ & p value \\
\hline CD56 & $0(0)$ & $0(1)$ & NS \\
CD57 & $1(0)$ & $1(0)$ & NS \\
MT & $1(1)$ & $0(1)$ & 0.03 \\
Vimentin & $2(1)$ & $1(1)$ & 0.005 \\
\hline
\end{tabular}

\section{CD57 antigen}

CD57-antigen immunoreactivity was detected in $95 \%$ of the lymphoma tissue samples and $85 \%$ of the stroma samples and represented the membranous-cytoplasmic type of expression (Fig. 6). CD57-antigen immunoreactivity was observed in all the samples of lymphatic tissue of the palatine tonsils under chronic inflammation and represented the membranous-cytoplasmic type of expression. It was not observed in the epithelium lining the tonsils (Fig. 6).

The comparison of analysed antigen immunoreactivity in the tissue of the lymphoma and its stroma is presented in Table 3.

A statistically significantly higher level of vimentin immunoreactivity was observed in the lymphoma tissue samples than in the stroma samples. Similarly, statistically significantly higher MT immunoreactivity was shown in the lymphoma than in the stroma tissue samples. No statistically significant differences were observed in CD56- and CD57-antigen immunoreactivity.

Table 4 shows the comparison of analysed antigen immunoreactivity in lymphatic tissue and the epithelium of the palatine tonsils of patients with chronic tonsillitis.

A statistically significantly higher MT immunoreactivity was observed in the epithelium lining the tonsils in comparison to the lymphatic tissue of the palatine tonsils from patients with chronic tonsillitis. Additionally, statistically significantly higher vimentin immunoreactivity was detected in the lymphatic tissue of palatine tonsils in comparison to the epithelium.

Table 5 shows the comparison of analysed antigen immunoreactivity in the lymphoma tissue samples and the lymphatic tissue samples from patients with chronic tonsillitis.

Statistically significantly higher MT immunoreactivity was observed in the lymphoma tissue samples than in the samples from the reference group (lymphatic tissue 
Table 4. The comparison of the analysed antigen immunoreactivity between the lymphatic tissue and epithelium lining the palatine tonsils from patients with chronic tonsillitis

\begin{tabular}{lccc}
\hline Antigen & $\begin{array}{c}\text { Lymphatic tissue of the palatine } \\
\text { tonsils } \\
\text { Median (Q3-Q1) }\end{array}$ & $\begin{array}{c}\text { Epithelium of the tonsils } \\
\text { Median (Q3-Q1) }\end{array}$ & $p$ value \\
\hline CD56 & $0(1)$ & $0(0)$ & 0.02 \\
CD57 & $3(1)$ & $0(0)$ & $p<0.0001$ \\
MT & $1(1)$ & $2(1)$ & 0.03 \\
Vimentin & $3(0)$ & $0(0)$ & $p<0.0001$ \\
\hline
\end{tabular}

Table 5. The comparison of analysed antigen immunoreactivity in the lymphoma tissue samples and in the lymphatic tissue of patients with chronic tonsillitis

\begin{tabular}{lccc}
\hline Antigen & $\begin{array}{c}\text { Lymphoma } \\
\text { Median (O3-Q1) }\end{array}$ & $\begin{array}{c}\text { Lymphatic tissue of palatine tonsils } \\
\text { under chronic tonsillitis } \\
\text { Median (Q3-Q1) }\end{array}$ & $\mathbf{p}$ value \\
\hline CD56 & $0(0)$ & $0(1)$ & $\mathrm{NS}$ \\
CD57 & $1(0)$ & $3(1)$ & $\mathrm{p}<0.001$ \\
MT & $2(1)$ & $1(1)$ & 0.03 \\
Vimentin & $2(1)$ & $3(0)$ & $\mathrm{p}<0.001$ \\
\hline
\end{tabular}

Table 6. The comparison of the analysed antigen immunoreactivity in the stroma and in the epithelium lining the palatine tonsils from patients with chronic tonsillitis.

\begin{tabular}{lccc}
\hline Antigen & $\begin{array}{c}\text { Stroma } \\
\text { Median (O3-Q1) }\end{array}$ & $\begin{array}{c}\text { Epithelium lining the palatine tonsils } \\
\text { under chronic tonsillitis } \\
\text { Median (Q3-Q1) }\end{array}$ & $p$ value \\
\hline CD56 & $0(1)$ & $0(0)$ & 0.005 \\
CD57 & $1(0)$ & $0(0)$ & $p<0.001$ \\
MT & $0(1)$ & $2(1)$ & $p<0.001$ \\
Vimentin & $1(1)$ & $0(0)$ & $p<0.001$ \\
\hline
\end{tabular}

of palatine tonsils from patients with chronic tonsillitis). Additionally, statistically significantly higher vimentin immunoreactivity was observed in the lymphoma tissue samples than in the reference group samples.

Table 6 shows the comparison of analysed antigen immunoreactivity in the stroma and the epithelium lining the palatine tonsils of patients with chronic tonsillitis.

Statistically significantly higher immunoreactivity of CD56, CD57, and vimentin antigens was observed in the stroma of the tumour than in the reference group samples. Additionally, statistically significantly higher MT immunoreactivity was detected in the reference group samples than in the tumour stroma samples.

\section{Discussion}

In the present analysis, MT immunoreactivity was demonstrated in the lymphoma cells and was statistical- ly significantly higher in the tumour tissue samples than in the stroma samples and in the tumour tissue samples compared to the reference samples. Poulsen et al. found MT expression on DLBCL lymphoma cells. They also identified a correlation between MT expression and prognosis. Specifically, MT labelling of more than $20 \%$ lymphoma cells was associated with a significantly poorer five-year survival rate, independent of the age, stage, or International Prognostic Index. It was thus suggested that both increased MT mRNA and MT protein expression by more than $20 \%$ of lymphoma cells are independent risk factors for DLBCL [39]. MT is a protein that both protects cells against apoptosis and induces cell proliferation. MT expression by malignant cells may thus have two important consequences: inducing the proliferation of malignant cells and developing resistance to the apoptosis stimulated by immune system cells. Both of these phenomena enable tumour growth and the development of the disease [14]. 
In the present study, MT immunoreactivity was also identified in the tumour stroma samples and was significantly lower than in the lymphoma samples. This sole MT immunoreactivity presence in the stroma samples is intriguing. In the present study, MT immunoreactivity was also found to be expressed by the stromal fibroblasts. This correlates with the pattern of MT expression in other histological tumour types, such as adenocarcinomas and squamous cell carcinomas [22-25]. Stroma is tissue that undergoes intensive remodelling during tumour development, which is also induced by factors secreted from its own tumour cells [40]. This remodelling is realised according to the tumour scenario stimulating its growth. Fibroblasts differentiate into myofibroblasts, the basic stromal cells in remodelled stroma, which respond to tumour needs (carcinoma-associated fibroblasts or CAFs) [8, 13-14, 22, 23]. Moreover, in the present study, MT immunoreactivity was also observed on macrophages in the lymphoma tissue samples. This observation is in agreement with that of Poulsen et al. who showed MT to be present in macrophages and that the number of MT-positive macrophages did not correlate with the patient's survival [39]. Macrophages also play an important role in remodelling the tumour microenvironment [40]. Thus, MT may be a marker of stroma remodelling and the development of tumour-associated fibroblasts and macrophages. Numerous authors have also described the expression of MT by healthy cells. MT was present in the basal layer of the epithelium, which is responsible for renewal [14]. In a previous study, we presented MT expression in the stroma of both breast and head and neck carcinomas $[13,14,19,20]$. We concluded that an aggressive inflammatory infiltrate in the tumour may induce MT expression as a development of the resistance to the apoptosis stimulated by immune system cells $[13,14,19,20]$.

In the present study, vimentin immunoreactivity was found in all the lymphoma tissue samples and in $80 \%$ of the stroma tissue samples. It was significantly higher in the lymphoma samples than in the stroma samples, and higher again in the lymphoma samples than in the reference samples. Additionally, it was significantly higher in the stroma than in the reference samples. Vimentin is one of the markers of mesenchymal phenotype of cell acquisition linked with the epithelial-mesenchymal transition. This process is typified by the change in expression of epithelial markers to mesenchymal markers such as vimentin and $\mathrm{N}$-cadherin [35]. This process is also observed in physiology, for example during wound healing, but in pathology the mesenchymal phenotype is typical of metastasising cells [41, 42]. MT may therefore be together with vimentin the marker of stromal remodelling in relation to the epithelial-mesenchymal transition (EMT) phenomenon.
In the present study, the infiltration of CD56- and CD57-positive cells into the stroma and lymphoma remained at comparable levels. This correlated with our observation of the CD56- and CD57-positive cell infiltration into adenocarcinoma and its stroma [22]. However, a significantly lower infiltration of CD57-positive cells was observed in the lymphoma than in the reference samples. Thus, the lower level of anticancer response represented by CD56- and CD57-positive lymphocytes in the lymphoma and its stroma indicates a deficit of this response in the analysed lymphoma tissue.

In summary, the high vimentin immunoreactivity in the tumour and its stroma, together with the presence of MT-expressing fibroblasts and macrophages, would seem to confirm microenvironment remodelling as well as the participation of MT in the tumour remodelling process.

\section{References}

1. Doll DC. Introduction: extranodal lymphomas. Semin Oncol. 1999; 26: 249-250.

2. Jacobs C, Hoppe RT. Non-Hodgkin's lymphomas of head and neck extranodal sites. Int J Radiat Oncol Biol Phys. 1985; 11(2): 357-364, indexed in Pubmed: 3882644.

3. Jacobs C, Weiss L, Hoppe RT. The management of extranodal head and neck lymphomas. Arch Otolaryngol Head Neck Surg. 1986; 112(6): 654-658, indexed in Pubmed: 3516178.

4. Banfi A, Bonadonna G, Carnevali G, et al. Lymphoreticular sarcomas with primary involvement of Waldeyer's ring. Clinical evaluation of 225 cases. Cancer. 1970; 26(2): 341-351, indexed in Pubmed: 4916022.

5. Avilés A, Delgado S, Ruiz H, et al. Hodgkin's and non-Hodgkin's lymphoma. Eur J Cancer B Oral Oncol. 1996; 32B: 19-23, indexed in Pubmed: 8729614.

6. Harabuchi $\mathrm{Y}$, Tsubota H, Ohguro S, et al. Prognostic factors and treatment outcome in non-Hodgkin's lymphoma of Waldeyer's ring. Acta Oncol. 1997; 36(4): 413-420, indexed in Pubmed: 9247103.

7. Yamanaka N, Harabuchi Y, Sambe S, et al. Non-Hodgkin's lymphoma of Waldeyer's ring and nasal cavity. Clinical and immunologic aspects. Cancer. 1985; 56(4): 768-776, indexed in Pubmed: 4016670.

8. Cherian MG, Jayasurya A, Bay BH. Metallothioneins in human tumors and potential roles in carcinogenesis. Mutat Res. 2003; 533(1-2): 201-209, indexed in Pubmed: 14643421.

9. Kagi JH, Scheffer A. of metallothionein. Biochemistry of metallothionein Biochemistry. 2003; 27: 8509-8515.

10. Cherian MG, Apostolova MD. Nuclear localization of metallothionein during cell proliferation and differentiation. Cell Mol Biol (Noisy-legrand). 2000; 46(2): 347-356, indexed in Pubmed: 10774924.

11. Fan $L Z$, Cherian MG. Potential role of p53 on metallothionein induction in human epithelial breast cancer cells. Br J Cancer. 2002; 87(9): 1019-1026, doi: 10.1038/sj.bjc.6600549, indexed in Pubmed: 12434295.

12. Werynska B, Pula B, Kobierzycki $C$, et al. Metallothioneins in the lung cancer. Folia Histochemica et Cytobiologica. 2015; 53(1): 1-10, doi: 10.5603/fhc.a2015.0009

13. Dutsch-Wicherek M, Popiela TJ, Klimek M, et al. Metallothionein stroma reaction in tumor adjacent healthy tissue in head and neck squamous cell carcinoma and breast adenocarcinoma. Neuro Endocrinol Lett. 2005; 26(5): 567-574, indexed in Pubmed: 16264399.

14. Dutsch-Wicherek M, Sikora J, Tomaszewska R. The possible biological role of metallothionein in apoptosis. Front Biosci. 2008; 13: 4029-4038, indexed in Pubmed: 18508497

15. Hellquist HB. Apoptosis in epithelial hyperplastic laryngeal lesions. Acta Otolaryngol Suppl. 1997; 527: 25-29, indexed in Pubmed: 9197476.

16. Muramatsu $Y$, Hasegawa $Y$, Fukano $\mathrm{H}$, et al. Metallothionein immunoreactivity in head and neck carcinomas; special reference to clinical behaviors and chemotherapy responses. Anticancer Res. 2000; 20(1A): 257-264, indexed in Pubmed: 10769664.

17. Sundelin K, Jadner M, Norberg-Spaak L, et al. Metallothionein and Fas (CD95) are expressed in squamous cell carcinoma of the tongue. Eur J Cancer. 1997; 33(11): 1860-1864, indexed in Pubmed: 9470847. 
18. Theocharis SE, Margeli AP, Klijanienko JT, et al. Metallothionein expression in human neoplasia. Histopathology. 2004; 45(2): 103-118, doi: 10.1111/j.1365-2559.2004.01922.x, indexed in Pubmed: 15279628.

19. Popiela TJ, Rudnicka-Sosin L, Dutsch-Wicherek M, et al. The metallothionein and RCAS1 expression analysis in breast cancer and adjacent tissue regarding the immune cells presence and their activity. Neuro Endocrinol Lett. 2006; 27(6): 786-794, indexed in Pubmed: 17187007.

20. Popiela TJ, Klimek M, Wicherek L, et al. The characterization of the exposure to immune mediated apoptosis and the regulation of immune cytotoxic activity in the environment of a neoplasm and in decidua. Neuro Endocrinol Lett. 2006; 27(6): 779-785, indexed in Pubmed: 17187008

21. Witz I. The Tumor Microenvironment: The Making of a Paradigm Cancer Microenvironment. 2009; 2(S1): 9-17, doi: 10.1007/s12307009-0025-8.

22. Dutsch-Wicherek M, Lazar A, Tomaszewska R. The potential role of $\mathrm{mt}$ and vimentin immunoreactivity in the remodeling of the microenvironment of parotid adenocarcinoma. Cancer Microenviron. 2010 4(1): 105-113, doi: 10.1007/s12307-010-0058-z, indexed in Pubmed 21505565.

23. Dutsch-Wicherek M, Lazar A, Tomaszewska R, et al. Analysis of metallothionein and vimentin immunoreactivity in pharyngeal squamous cell carcinoma and its microenvironment. Cell Tissue Res. 2013; 352(2) 341-349, doi: 10.1007/s00441-013-1566-1, indexed in Pubmed: 23397427

24. Dutsch-Wicherek M, Tomaszewska R, Lazar A, et al. The evaluation of metallothionein expression in nasal polyps with respect to immune cell presence and activity. BMC Immunol. 2010; 11: 10, doi: 10.1186/1471 2172-11-10, indexed in Pubmed: 20214821.

25. Walentowicz-Sadlecka M, Koper A, Krystyna G, et al. The analysis of metallothionein immunoreactivity in stromal fibroblasts and macrophages in cases of uterine cervical carcinoma with respect to both the local and distant spread of the disease. Am J Reprod Immunol. 2013 70(3): 253-261, doi: 10.1111/aji.12120, indexed in Pubmed: 23590599

26. Kazmierczak W, Lazar A, Tomaszewska R, et al. Analysis of the intensity of immune cell infiltration and immunoreactivity of RCAS1 in diffuse large B-cell lymphoma of the palatine tonsil and its microenvironment. Cell Tissue Res. 2015; 361(3): 823-831, doi: 10.1007/s00441-0152157-0, indexed in Pubmed: 25773455

27. Franke WW, Grund C, Kuhn C et al. Formation of cytoskeletal elements during mouse embryogenesis. III. Primary mesenchymal cells and the first appearance of vimentin filaments. Differentiation. 1982; 23(1): 43-59, indexed in Pubmed: 6759279.

28. Larsson A, Wilhelmsson U, Pekna M, et al. Increased cell proliferation and neurogenesis in the hippocampal dentate gyrus of old GFAP(-/-) Vim(-/-) mice. Neurochem Res. 2004; 29(11): 2069-2073, indexed in Pubmed: 15662841.
29. Cochard P, Paulin D. Initial expression of neurofilaments and vimentin in the central and peripheral nervous system of the mouse embryo in vivo. J Neurosci. 1984; 4(8): 2080-2094, indexed in Pubmed: 6432971.

30. de Souza PC, Katz SG. Coexpression of cytokeratin and vimentin in mice trophoblastic giant cells. Tissue Cell. 2001; 33(1): 40-45, doi: 10.1054/tice.2000.0148, indexed in Pubmed: 11292169.

31. Ko SH, Suh SH, Kim BJ, et al. Expression of the intermediate filament vimentin in proliferating duct cells as a marker of pancreatic precursor cells. Pancreas. 2004; 28(2): 121-128, indexed in Pubmed: 15028943.

32. Mahrle G, Bolling R, Osborn M, et al. Intermediate filaments of the vimentin and prekeratin type in human epidermis. J Invest Dermatol. 1983; 81(1): 46-48, indexed in Pubmed: 6190951.

33. Carter V, Shenton BK, Jaques B, et al. Vimentin antibodies: a non-HLA antibody as a potential risk factor in renal transplantation. Transplant Proc. 2005; 37(2): 654-657, doi: 10.1016/j.transproceed.2004.12.043, indexed in Pubmed: 15848491

34. Evans RM. Vimentin: the conundrum of the intermediate filament gene family. Bioessays. 1998; 20(1): 79-86, doi: 10.1002/(SICI)15211878(199801)20:1 < 79::AID-BIES11 > 3.0.CO;2-5, indexed in Pubmed: 9504050.

35. Thiery JP. Epithelial-mesenchymal transitions in tumour progression. Nat Rev Cancer. 2002; 2(6): 442-454, doi: 10.1038/nrc822, indexed in Pubmed: 12189386.

36. Chaffer CL Brennan JP Slavin لال et al Mesenchymal-to-epithelial transition facilitates bladder cancer metastasis: role of fibroblast growth factor receptor-2. Cancer Res. 2006; 66(23): 11271-11278, doi: 10.1158/0008-5472.CAN-06-2044, indexed in Pubmed: 17145872

37. Satelli A, Li S. Vimentin in cancer and its potential as a molecular target for cancer therapy. Cell Mol Life Sci. 2011; 68(18): 3033-3046, doi: 10.1007/s00018-011-0735-1, indexed in Pubmed: 21637948

38. Imoto A, Okada M, Okazaki T, et al. Metallothionein-1 isoforms and vimentin are direct PU.1 downstream target genes in leukemia cells. J Biol Chem. 2010; 285(14): 10300-10309, doi: 10.1074/jbc. M109.095810, indexed in Pubmed: 20139074

39. Poulsen CB, Borup R, Borregaard N, et al. Prognostic significance of metallothionein in B-cell lymphomas. Blood. 2006; 108(10): 3514-3519, doi: 10.1182/blood-2006-04-015305, indexed in Pubmed: 16868254.

40. Mantovani A, Allavena P, Sica A, et al. Cancer-related inflammation. Nature. 2008; 454(7203): 436-444, doi: 10.1038/nature07205, indexed in Pubmed: 18650914

41. Hugo $\mathrm{H}$, Ackland ML, Blick T, et al. Epithelial--mesenchymal and mesenchymal-epithelial transitions in carcinoma progression. J Cell Physiol. 2007 213(2): 374-383, doi: 10.1002/jcp.21223, indexed in Pubmed: 17680632

42. Grünert S, Jechlinger $M$, Beug $H$. Diverse cellular and molecular mechanisms contribute to epithelial plasticity and metastasis. Nat Rev Mol Cell Biol. 2003; 4(8): 657-665, doi: 10.1038/nrm1175, indexed in Pubmed: 12923528 
